\title{
Performances bioéconomiques des poulettes alimentées avec des rations à base de feuilles séchées de manioc (Manihot esculenta)
}

\author{
M. F. HOUNDONOUGBO*, C. A. A. M. CHRYSOSTOME et V. P. HOUNDONOUGBO \\ Laboratoire de Recherche Avicole et de Zoo-Economie, Département de Production animale, \\ Faculté des Sciences Agronomiques, l'Université d'Abomey-Calavi, 01 BP 526 Cotonou, République du Bénin. \\ *Auteur correspondant, E-mail : fredericmh@gmail.com; Tel. : (+229) 96142965 ou (+229) 95968136
}

\section{RESUME}

L'efficience des rations alimentaires à base de feuilles séchées de manioc doux a été évaluée en alimentation des poulettes pendant seize semaines. Trois rations contenant $0 \%$ (R0), 5\% (R5) et 10\% (R10) de feuilles séchées de manioc ont été utilisées pour nourrir 225 poussins d'un jour de souche ISA Brown répartis en groupe de 25 poussins avec trois répétitions par ration. Les gains de poids moyen quotidien $(8,5$ à $9,2 \mathrm{~g} / \mathrm{j})$ et les indices de consommation ( $\mathrm{IC}=7,0$ à 7,58) sont similaires $(\mathrm{P}>0,05)$ pour toutes les rations alimentaires. L'incorporation des feuilles, n'a pas empêché d'atteindre les besoins nutritionnels recommandés aux poulettes. Ainsi, les rations à base de feuilles séchées de manioc, n'ont pas influé sur le taux de mortalité des poulettes. Mais, elles ont permis de réduire significativement $(\mathrm{P}<0,05)$ le coût alimentaire. Par rapport à la ration R0, le coût alimentaire global a baissé de $11,5 \%$ et $7,4 \%$ respectivement pour les rations R5 et R10. Sur les deux phases expérimentales, les meilleures performances bioéconomiques ont été enregistrées chez les poulettes ayant reçu la ration contenant $5 \%$ de feuilles.

(C) 2012 International Formulae Group. All rights reserved.

Mots clés : Gain de poids, indice de consommation, taux optimal, coût alimentaire.

\section{INTRODUCTION}

Les ressources alimentaires non conventionnelles peu ou pas valorisées en alimentation de volaille, peuvent constituer des ingrédients alternatifs à moindre coût. Les feuilles de manioc par exemple sont riches en protéines $(21 \%)$, en carotènes, en minéraux et en vitamines $\mathrm{B} 1$, B2, et $\mathrm{C}$; mais leur contenance en cyanure et la pauvreté de leurs protéines en acides aminés soufrés (méthionine et cystine) limitent leur large utilisation (Ravindran, 1993). Toutefois, un séchage au soleil réduit significativement la cyanure des feuilles et diminue leur taux de tanin (Eruvbetine et al., 2003).

Chez les poulets de chair, des baisses de gain de poids et d'efficience alimentaire sont signalées en cas d'incorporation de feuilles dans l'aliment (Eruvbetine et al., 2003 ; Fasuyi et Aletor, 2005). Toutefois, D'Mello (1995) recommandait 5\% de farine de feuilles séchées dans l'aliment des poulets de chair. En élevage de pintade, Dahouda et al. (2009) rapportaient que 25 à $35 \%$ de cossettes et 7 à $8 \%$ de feuilles de manioc pouvaient constituer des sources alternatives d'énergie et de protéines, en particulier au 
cours de la période de finition. Plus tôt, Odunsi et al. (1999) avaient démontré qu'il y a une amélioration de la marge bénéficiaire en élevage de volaille en cas d'utilisation d'aliments contenant des feuilles de végétaux.

Une expérimentation a été donc conduite en vue d'évaluer dans les conditions tropicales du Bénin, les performances de croissance pondérale des poulettes alimentées avec des rations contenant différents taux de feuilles séchées de manioc doux.

\section{MATERIEL ET METHODES Dispositif expérimental}

Un total de 225 poussins d'un jour de la souche ISA Brown était réparti en groupe de 25 poussins. Ils ont été nourris de façon $a d$ libitum avec trois rations R0, R5 et R10 contenant respectivement 0,5 et $10 \%$ de feuilles séchées de manioc doux avec trois répétitions. Ces taux de feuilles ont été adoptés aux phases poussins et poulette d'égales durées de 8 semaines.

Les rations ont été fabriquées en incorporant directement les feuilles séchées de manioc doux dans le mélange farineux réalisé dans une fabrique d'aliment. La composition des rations à différentes phases d'élevage est présentée dans le Tableau 1.

Les poulettes étaient élevées au sol sur litière. Elles étaient pesées hebdomadairement. Les consommations d'aliment étaient évaluées quotidiennement. En se basant d'une part, sur le coût de la main d'œuvre pour la récolte et le séchage, et d'autre part, sur les charges de transport, la valeur économique des feuilles séchées de manioc a été estimée à $50 \mathrm{FCFA} / \mathrm{kg}$.

\section{Analyses statistiques}

Les variables issues des données collectées ont été analysées dans le logiciel SAS version 9.1 (2004) par la procédure du General Linear Model (GLM) selon le model suivant :

$\mathrm{Y}_{\mathrm{i}}=\mu+\mathrm{R}_{\mathrm{i}}+\varepsilon_{\mathrm{i}}$
$Y_{i}=$ Observation des variables dépendantes

$\mu=$ moyenne générale

$\mathrm{R}_{\mathrm{i}}=$ Effet fixe de la ration alimentaire

$\varepsilon_{\mathrm{i}}=$ Erreur résiduelle

Les valeurs moyennes des variables ont été présentées dans des tableaux avec les probabilités (P) issues de leur comparaison. L'effet de la ration alimentaire est dit significatif si $\mathrm{P}<0,05$.

\section{RESULTATS}

Ingestion et efficacité alimentaire des poulettes

L'ingestion d'aliment par les poulettes était similaire $(\mathrm{P}>0,05)$ pour les trois rations R0, R5 et R10 au cours des deux phases d'élevage (Tableau 2). L'incorporation des feuilles séchées de manioc jusqu'à $10 \%$ dans la ration des poulettes n'a donc pas affecté significativement leur ingestion d'aliment.

Aux cours des deux phases, l'indice de consommation alimentaire (IC) des poulettes était faible pour la ration $\mathrm{R} 5$, sans que la différence avec les IC des deux autres rations ne soit significative $(P>0,05)$. Par rapport aux poulettes des rations $\mathrm{R} 0$ et $\mathrm{R} 10$, les poulettes ayant reçu la ration contenant $5 \%$ de feuilles séchées de manioc ont donc mieux valorisé l'aliment. Indépendamment de la ration, l'ingestion et l'indice de consommation alimentaire ont augmenté de la phase poussin à celle poulette (Tableau 2). L'efficacité alimentaire des poulettes a donc diminué avec l'âge.

\section{Croissance pondérale et mortalité des poulettes}

De 0 à 7 semaines d'âge, la croissance était semblable pour les trois rations (Figure 1). Après cet âge, la croissance pondérale était meilleure chez les poulettes nourries avec la ration contenant $5 \%$ de feuilles de manioc.

Par contre, l'incorporation de $10 \%$ de feuilles de manioc a altéré la croissance pondérale des poulettes sans que les gains moyens quotidiens des trois rations (Tableau 
3) ne soient significativement différents $(\mathrm{P}>$ 0,05).

A seize semaines d'âge, les poids vif des poulettes soumises aux rations R0, R5 et $\mathrm{R} 10$ étaient respectivement de $1053 \mathrm{~g}, 1080 \mathrm{~g}$ et $994 \mathrm{~g}$. Par ailleurs, l'effet des rations sur le taux de mortalité des poulettes n'était pas significatif $(\mathrm{P}>0,05)$. En effet, durant l'essai, un seul poussin était mort dans le traitement R5 (soit 1,33\%) contre zéro dans les rations $\mathrm{R} 0$ et R10.

\section{Coût alimentaire des poulettes}

L'utilisation des feuilles séchées de manioc a permis de réduire le prix des aliments destinés aux poussins et aux poulettes, respectivement de 31 à 38 FCFA/kg et de 11 à $17 \mathrm{FCFA} / \mathrm{kg}$ par rapport à la ration témoin R0 (Tableau 4). Cette réduction s'est donc accrue avec l'augmentation du taux de feuilles dans l'aliment. En conséquence, par rapport à la ration témoin, le coût alimentaire chez les poulettes nourries avec les rations à base de feuilles était significativement plus faible aux deux phases d'élevage. Cette tendance était plus remarquable pendant les deux premiers mois qui correspondaient à la phase de démarrage des poussins. En effet, par rapport à la ration témoin $\mathrm{R} 0$, les rations $\mathrm{R} 5$ et R10 ont permis de réduire, le coût alimentaire, respectivement de, $17 \%$ et $11 \%$ à la phase poussin contre $8,2 \%$ et $5,5 \%$ à la phase poulette. Sur le plan économique, les deux taux d'incorporation de feuilles séchées de manioc, étaient donc acceptables en alimentation des poulettes avec toutefois une ascendance pour le taux de $5 \%$.

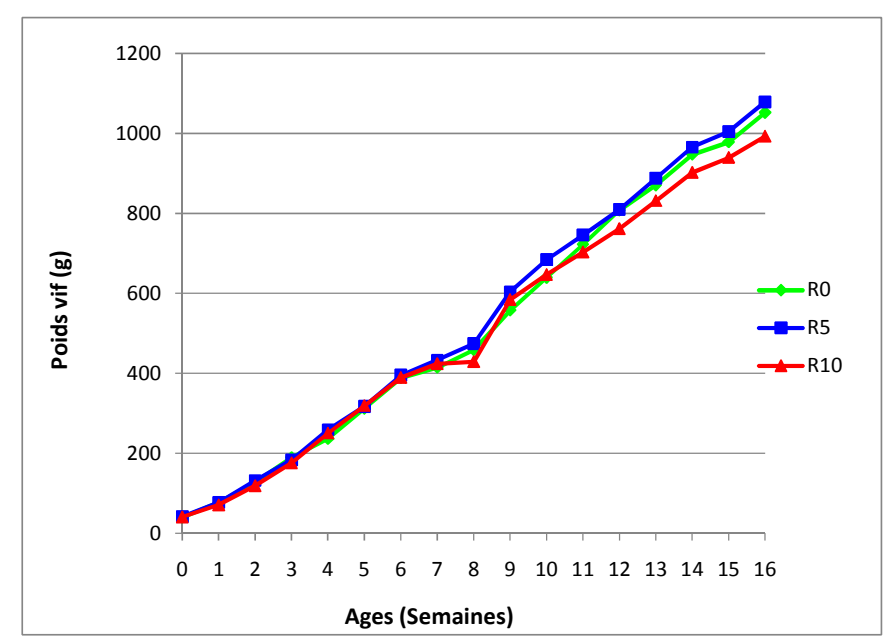

Figure 1: Evolution de la croissance pondérale des poulettes ISA Brown nourries avec des rations à base de feuilles séchées de manioc doux. 
Tableau 1: Composition des aliments expérimentaux en ingrédients et en nutriments.

\begin{tabular}{|c|c|c|c|c|c|c|}
\hline \multirow[b]{2}{*}{ Ingrédients (\%) } & \multicolumn{3}{|c|}{$\begin{array}{l}\text { Phase poussin } \\
(0-8 \text { semaines })\end{array}$} & \multicolumn{3}{|c|}{$\begin{array}{l}\text { Phase poulette } \\
\text { (9-16 semaines) }\end{array}$} \\
\hline & R0 & R5 & R10 & R0 & R5 & R10 \\
\hline Feuilles séchées de manioc doux & 0 & 5 & 10 & 0 & 5 & 10 \\
\hline Maïs & 61,8 & 60 & 56 & 65 & 62,6 & 62,56 \\
\hline Son de blé & 5 & 5 & 5 & 9,6 & 9 & 7 \\
\hline Tourteau soja & 26 & 23 & 22 & 14 & 12 & 10 \\
\hline Tourteau coton & 4 & 4 & 4 & 7 & 7 & 6 \\
\hline Huile rouge & & & & 1 & 1 & 1 \\
\hline Coquille d'huile & 1,8 & 1,6 & 1,6 & 1,7 & 1,7 & 1,7 \\
\hline Lysine & 0,1 & 0,1 & 0,1 & 0,1 & 0,1 & 0,14 \\
\hline Méthionine & 0,1 & 0,1 & 0,1 & 0,1 & 0,1 & 0,1 \\
\hline Phosphate bicalcique & 0,7 & 0,7 & 0,7 & 1 & 1 & 1 \\
\hline Sel (Nacl) & 0,3 & 0,3 & 0,3 & 0,3 & 0,3 & 0,3 \\
\hline Prémix ${ }^{1}$ & 0,2 & 0,2 & 0,2 & 0,2 & 0,2 & 0,2 \\
\hline \multicolumn{7}{|l|}{ Eléments nutritifs } \\
\hline Matière Sèche, \% & 87,1 & 83,9 & 80,9 & 86,9 & 83,8 & 80,7 \\
\hline Cellulose brute, $\%$ & 4,28 & 4,59 & 5,00 & 4,30 & 4,61 & 4,71 \\
\hline Energie Métabolisable, kcal/kg MS & 2827 & 2835 & 2819 & 2857 & 2861 & 2906 \\
\hline Protéine brute, $\%$ & 18,9 & 18,7 & 19,1 & 15,9 & 15,9 & 15,7 \\
\hline Lysine, \% & 1,02 & 0,94 & 0,90 & 0,78 & 0,72 & 0,67 \\
\hline Méthionine, \% & 0,41 & 0,39 & 0,38 & 0,37 & 0,35 & 0,33 \\
\hline Acide aminé soufré, \% & 0,74 & 0,70 & 0,67 & 0,66 & 0,62 & 0,57 \\
\hline Calcium, \% & 0,93 & 0,94 & 1,05 & 0,91 & 1,01 & 1,11 \\
\hline Phosphore total, \% & 0,60 & 0,66 & 0,73 & 0,67 & 0,73 & 0,77 \\
\hline
\end{tabular}

Tableau 2: Ingestion d'aliment et Indice de consommation alimentaire (IC) des poulettes nourries avec des rations à base de feuilles séchées de manioc doux.

\begin{tabular}{lcccccccc}
\hline & \multicolumn{4}{c}{ Ingestion d'aliment (g/poulette/j) } & \multicolumn{3}{c}{ IC (g aliment/kg gain poids vif) } \\
\hline Phases & R0 & R5 & R10 & Probabilité & R0 & R5 & R10 & Probabilité \\
\hline Poussins & 44,1 & 43,7 & 43,4 & 0,43 & 5,96 & 5,71 & 6,34 & 0,41 \\
Poulettes & 86,4 & 85,6 & 84,9 & 0,35 & 8,16 & 7,92 & 8,42 & 0,38 \\
Globale & 60,9 & 60,3 & 59,9 & 0,49 & 7,25 & 7,01 & 7,58 & 0,55 \\
\hline
\end{tabular}


Tableau 3: Gain moyen quotidien de poids vif $(\mathrm{g} / \mathrm{j})$ des poulettes ISA Brown nourries avec des rations à base de feuilles séchées de manioc doux.

\begin{tabular}{lcccc}
\hline Phases & R0 & R5 & R10 & Probabilité \\
\hline Poussins & 7,40 & 7,65 & 6,85 & 0,47 \\
Poulettes & 10,6 & 10,8 & 10,1 & 0,55 \\
Globale & 9,00 & 9,22 & 8,46 & 0,43 \\
\hline
\end{tabular}

Tableau 4 : Prix des aliments et coût alimentaire chez les poulettes ISA Brown nourries avec des rations à base de feuilles séchées de manioc doux.

\begin{tabular}{lccccc}
\hline & Phases & R0 & R5 & R10 & Probabilité \\
\hline Prix des aliments & Poussins & 232 & 201 & 194 & - \\
(FCFA /kg aliment) & Poulettes & 201 & 190 & 184 & - \\
\hline \multirow{2}{*}{ Coût alimentaire } & Poussins & $1382^{\mathrm{a}}$ & $1149^{\mathrm{b}}$ & $1230^{\mathrm{c}}$ & 0,02 \\
(FCFA aliment /kg de gain de poids) & Poulettes & $1640^{\mathrm{a}}$ & $1506^{\mathrm{b}}$ & $1550^{\mathrm{b}}$ & 0,04 \\
& Globale & $1534^{\mathrm{a}}$ & $1358^{\mathrm{b}}$ & $1420^{\mathrm{c}}$ & 0,03 \\
\hline
\end{tabular}

Les moyennes suivies de différentes lettres sur la même ligne sont significativement différentes $(\mathrm{P}<0.05)$.

\section{DISCUSSION}

Qualité des aliments et efficacité alimentaire des poulettes

Les trois rations expérimentales sont presque iso-énergétiques et iso-protéiques. De plus, le taux de cellulose brute est maintenu entre 4 et $5 \%$ dans les rations. Mais, l'incorporation de feuilles séchées de manioc a engendré une légère baisse du taux de lysine, de méthionine et d'acides aminés soufrés. Ceci confirme la pauvreté des feuilles de manioc en acides aminés (Ravindran, 1993 ; Agunbiade et al., 2002). Toutefois, le niveau de ces acides aminés essentiels est maintenu tout au moins aux taux recommandés par INRA (1989) et NRC (1994). Par contre, les taux de calcium et de phosphore total se sont améliorés dans les rations R5 et R10 contenant des feuilles de manioc. Enfin, avec l'incorporation des feuilles de manioc, le prix du kg d'aliment a baissé de 13 à $16 \%$ et de 5 à $9 \%$ respectivement aux phases poussins et poulettes par rapport aux prix des aliments témoins (R0). L'utilisation des feuilles séchées de manioc n'entraine donc pas des contraintes nutritionnelles et économiques majeures pour la formulation des rations alimentaires de poulettes.

La similarité d'ingestion alimentaire (IA) notée chez les poulettes nourries avec les trois rations indique une bonne appétence des trois aliments. Cette similarité peut être aussi liée à l'atteinte de la capacité d'ingestion des poulettes à chaque phase. En effet, les IA enregistrées à la phase poussins sont très supérieures aux valeurs (22,9 et $23,7 \mathrm{~g}$ ) obtenues chez des poulettes de la souche Black Nera (0 à 6 semaines d'âge) nourries avec des rations contenant 0 à $6 \%$ des feuilles séchées de Centrosema pubescens (Nworgu et Fasogbon, 2007). De même, les quantités d'aliment ingérées à la phase poulette sont plus élevées que le maximum de $67 \pm 2,5 \mathrm{~g} / \mathrm{j}$ obtenu chez des poulettes de 17 semaines d'âge rapporté par Mutayoba et al. (2003).

Ainsi, à la phase poussin, les indices de consommation de cette étude représentent 
environ 1,5 à 2 fois ceux indiqués par Nworgu et Fasogbon (2007); alors qu'à la phase poulette nos valeurs sont plus faibles que celles signalées $(8,44$ à $9,09 \mathrm{~g}$ d'aliment/g de gain de poids) par les mêmes auteurs.

\section{Croissance et efficience alimentaire des poulettes}

La disponibilité de feuilles de manioc à bon marché et sa bonne valeur nutritionnelle ont permis de réduire dans les aliments, le taux de maïs entre 1,8 et $5,8 \%$, le taux de tourteau de soja entre 2 et $4 \%$ et le taux de tourteau de coton de $1 \%$ dans l'aliment poulette spécifiquement. Il en résulte une réduction du prix des aliments $\mathrm{R} 5$ et $\mathrm{R} 10$ qui en combinaison avec la similarité des gains moyens quotidiens (GMQ) a engendré une diminution significative du coût alimentaire par $\mathrm{kg}$ vif de poulette produite. Les coûts alimentaires obtenus avec les rations R0, R5 et R10 sont inferieurs aux 2005 FCFA d'aliment $/ \mathrm{kg}$ de gain de poids vif enregistrés au Nigéria chez des poulettes (Nworgu et Fasogbon, 2007). Ceci témoigne de l'efficience des rations alimentaires à base de feuilles séchées de manioc doux aux deux phases d'élevage des poulettes.

Les GMQ de cette expérimentation sont proches des 5,57 à $8,07 \mathrm{~g} / \mathrm{j}$ et 12,48 à $13,21 \mathrm{~g} / \mathrm{j}$ rapportés, respectivement, à la phase poussin et à celle poulette (Nworgu et Fasogbon, 2007). Au cours de l'expérimentation, les GMQ obtenus (phase globale) sont meilleurs à ceux signalés $(6,82$ à 7,59 g/j) par Sogunle et al. (2009). Cependant, à la fin de l'essai, les poids vifs des poulettes $(1053 \mathrm{~g}, 1080 \mathrm{~g}$ et 994 $\mathrm{g}$, respectivement, dans R0, R5 et R10) sont inférieurs au poids moyen de $1120 \mathrm{~g}$ obtenus au même âge chez des poulettes black Bovan Nera (Amaefule et al., 2006). Cette différence de poids vif serait liée aux souches de poulets utilisées. Les taux de certains facteurs antinutritionnels comme l'acide cyanhydrique et le tanin baissent significativement dans les feuilles lors du séchage (Eruvbetine et al., 2003). La composition nutritionnelle et le taux de cellulose brute étant similaires dans les rations, la baisse de performance chez les poulettes ayant reçu la ration $\mathrm{R} 10$ pourrait être liée à l'effet de résidus d'acide cyanhydrique et de tanin dans les feuilles séchées de manioc comme l'ont signalé Agunbiade et al. (2002) et Fasuyi et Aletor (2005).

Les résultats de cette étude sont comparables à ceux de Mutayoba et al. (2003) qui ont démontré que les feuilles séchées de Leucaena leucocephala incorporées à 5\% dans la ration des poulettes n'avaient pas d'effet adverse sur leur croissance; ce qui n'était pas le cas lorsque ce taux passe à $10 \%$ à cause du taux élevé de cellulose brute et de la présence de mimosine. Ainsi, conformément aux recommandations de D'Mello (1995), des aliments contenant 5\% de feuilles de manioc permettent de maintenir l'ingestion alimentaire et les performances de croissance pondérale des poulettes à des niveaux similaires à ceux des poulettes alimentées avec une ration témoin dépourvue de feuilles, et ceci, sans un effet significatif sur la mortalité qui s'est limitée à un seul poussin dans R5.

En somme, les feuilles séchées de manioc doux constituent des ingrédients pouvant substituer partiellement à un taux de $5 \%$, les sources conventionnelles d'énergie et de protéine dans l'aliment des poulettes. Leurs effets sur la production et la qualité des œufs méritent aussi d'être évalués chez les poules pondeuses.

\section{REFERENCES}

Agunbiade JA, Adeyemi OA, Adepoju OA, Lawal OA. 2002. The use of whole cassava meal and leaf meal in broiler diets. Tropical Journal of Animal Sciences, 5: 161-173.

Amaefule KU, Ojewola GS, Ironkwe MC. 2006. Pigeon pea (Cajanus cajan) seed meal as protein source for pullets: response of pullets to higher inclusion level and prolonged feeding of raw or processed pigeon pea seed meal diets. International Journal of Poultry Science, 4(3): 289-295. 
Dahouda M, Toleba SS, Youssao AKI, Mama Ali AA, Ahounou S, Hornick JL. 2009. Utilisation des cossettes et des feuilles de manioc en finition des pintades (Numida meleagris): performances zootechniques, coûts de production, caractéristiques de la carcasse et qualité de la viande. Annale de Médecine Vétérinaire, 153: 82-87.

D’Mello JPF. 1995. Leguminous leaf meals in non-ruminant nutrition. In Tropical Legumes in Animal Nutrition. A.B International (1st edn). Wallingford: Oxon UK; 247-280.

Eruvbetine D, Tajudeen ID, Adeosun AT, Olojedde AA. 2003. Cassava (Manihot esculenta) leaf and tuber concentrate in diet for broiler chickens. Bioresources Technology, 86: 277-281.

Fasuyi AO, Aletor VA. 2005. Protein replacement value of cassava (Manihot esculenta, Crantz) leaf protein concentrate (CLPC) in broiler starter: Effect on performance, muscle growth, hematology and serum metabolites. International Journal of Poultry Science, 4(5): 339-349.

INRA. 1989. (Institut National de la Recherche Agronomique). Alimentation des Animaux Monogastriques: Porc, Lapin, Volailles $\left(2^{\text {nd }}\right.$ edn, révisée et corrigée). INRA : France.

Mutayoba SK, Mutayoba BM, Okot P. 2003. The performance of growing pullets fed diets with varying energy and leucaena leaf meal levels. Livestock Research for Rural Development, 15(8): article 2.

National Research Council (NRC). 1994. Nutrient Requirements of Poultry (9th Revised edn). National Academy Press: USA.

Nworgu FC, Fasogbon FO. 2007. Centrosema (Centrosema pubescens) Leaf meal as protein supplement for pullet chicks and growing pullets. International Journal of Poultry Science, 6(4): 255-260.

Odunsi AA, Farinu GO, Akinola JO, Togun VA. 1999. Growth, carcass characteristics and body composition of broiler chicks fed with sunflower composition and nutritive value of leaf protein (Tithonia diversifolia) forage meal. Tropical Animal Production Investigation, 2: 205211.

Ravindran V. 1993. Cassava leaves as animal feed: Potential and limitations. Journal of the Science of Food and Agriculture, 2: 141-150.

Statistic Analysis System (SAS). 2004. User's Guide Statistic: Analysis System Procedure Version 9.1.2. SAS Institute Inc. Cary, NC: USA.

Sogunle OM, Fanimo AO, Abiola SS, Bamgbose AM. 2009. Performance of growing pullets fed cassava peel meal diet supplemented with cashew nut reject meal. Archivos de Zootecnia, 58(221): 23-31. 